\title{
Neural signatures of arbitration between Pavlovian and instrumental action selection
}

\author{
Samuel J. Gershman, ${ }^{1,2, *}$ Marc Guitart-Masip, ${ }^{3,4}$ James F. Cavanagh ${ }^{5}$ \\ ${ }^{1}$ Department of Psychology and Center for Brain Science, Harvard University \\ ${ }^{2}$ Center for Brains, Minds and Machines \\ ${ }^{3}$ Max Planck-UCL Centre for Computational Psychiatry and Ageing Research \\ ${ }^{4}$ Aging Research Center, Karolinska Institute \\ ${ }^{5}$ Department of Psychology, University of New Mexico \\ *correspondence: gershman@fas.harvard.edu
}

June 2, 2020

\begin{abstract}
Pavlovian associations drive approach towards reward-predictive cues, and avoidance of punishment-predictive cues. These associations "misbehave" when they conflict with correct instrumental behavior. This raises the question of how Pavlovian and instrumental influences on behavior are arbitrated. We test a computational theory according to which Pavlovian influence will be stronger when inferred controllability of outcomes is low. Using a model-based analysis of a Go/NoGo task with human subjects, we show that theta-band oscillatory power in frontal cortex tracks inferred controllability, and that these inferences predict Pavlovian action biases. Functional MRI data revealed an inferior frontal gyrus correlate of action probability and a ventromedial prefrontal correlate of outcome valence, both of which were modulated by inferred controllability.
\end{abstract}




\section{Introduction}

Approaching reward-predictive stimuli and avoiding punishment-predictive stimuli are useful heuristics adopted by many animal species. However, these heuristics can sometimes lead animals astray - a phenomenon known as "Pavlovian misbehavior" [1, 2]. For example, reward-predictive stimuli invigorate approach behavior even when such behavior triggers withdrawal of the reward $[3,4]$, or the delivery of punishment $[5,6]$. Likewise, punishmentpredictive stimuli inhibit approach behavior even when doing so results in reduced net reward $[7,8,9]$.

A venerable interpretation of these and related findings is that they arise from the interaction between Pavlovian and instrumental learning processes [10]. The two-process interpretation has been bolstered by evidence from neuroscience that Pavlovian and instrumental influences on behavior are (at least to some extent) segregated anatomically [11]. In particular, the dorsal subdivision of the striatum (caudate and putamen in primates) is more closely associated with instrumental learning, whereas the ventral subdivision (nucleus accumbens) is more closely associated with Pavlovian learning [12, 13].

Any multi-process account of behavior naturally raises the question of arbitration: what decides the allocation of behavioral control to particular processes at any given point in time? One way to approach this question from a normative perspective is to analyze the computational trade-offs realized by different processes. The job of the arbitrator is to determine which process achieves the optimal trade-off for a particular situation. This approach has proven successful in understanding arbitration between different instrumental learning processes $[14,15,16,17]$. More recently, it has been used to understand arbitration between Pavlovian and instrumental processes [18]. The key idea is that instrumental learning is more statistically flexible, in the sense that it can learn reward predictions that are both action-specific and stimulus-specific, whereas Pavlovian learning can only learn stimulusspecific predictions. The cost of this flexibility is that instrumental learning is more prone to over-fitting: for any finite amount of data, there is some probability that the learned predictions will generalize incorrectly in the future, and this probability is larger for more flexible models, since they have more degrees of freedom with which to capture noise in the data.

The account sketched above can be formalized in terms of Bayesian model comparison [18]. Several implications follow. First, the Bayesian arbitration mechanism preferentially allocates control to the Pavlovian process initially, when there are less data and hence less support for the more flexible model. This is broadly consistent with the finding that the Pavlovian bias on instrumental responding declines with the amount of instrumental training [19]. Second, this initial preference should be stronger in relatively less controllable environments, where little predictive power is gained by conditionalizing predictions on action. Accordingly, Pavlovian bias increases with the amount of Pavlovian training [19].

Dorfman and Gershman [18] tested the controllability prediction more directly using a variant of the Go/NoGo paradigm, which has been widely employed as an assay of Pavlovian bias in human subjects $[22,23,24,21,20,25]$. This task crosses valence (winning reward vs. avoiding punishment) with action (Go vs. NoGo), resulting in four conditions: Go- 
A

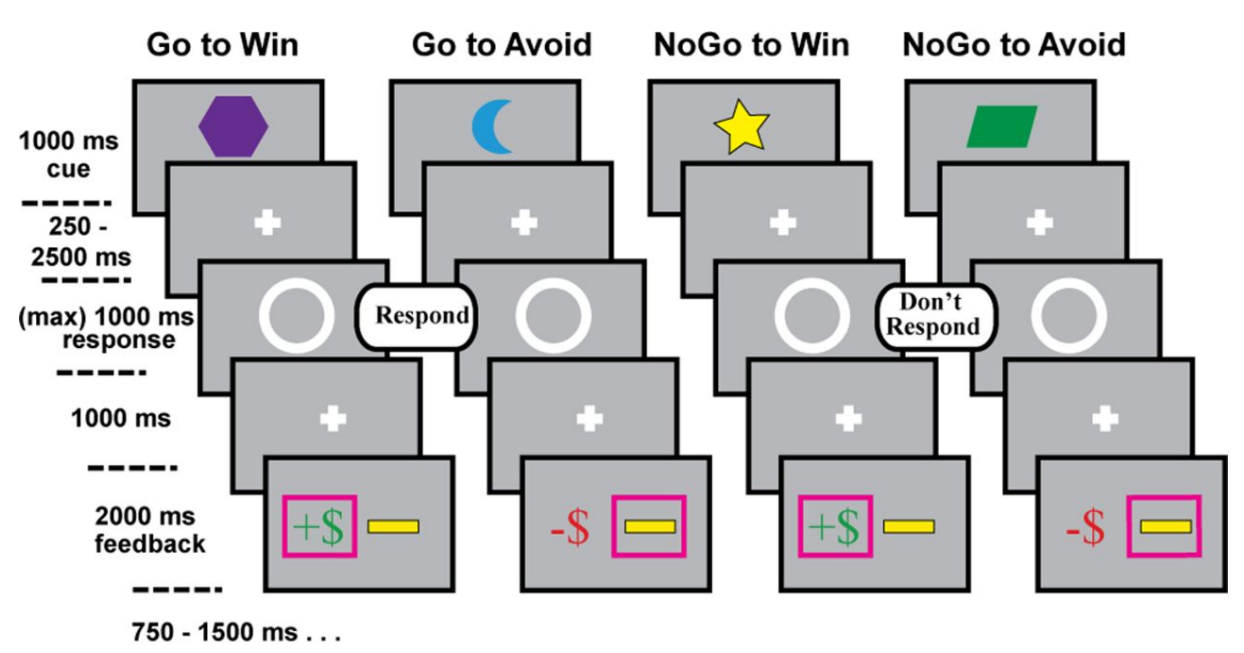

B

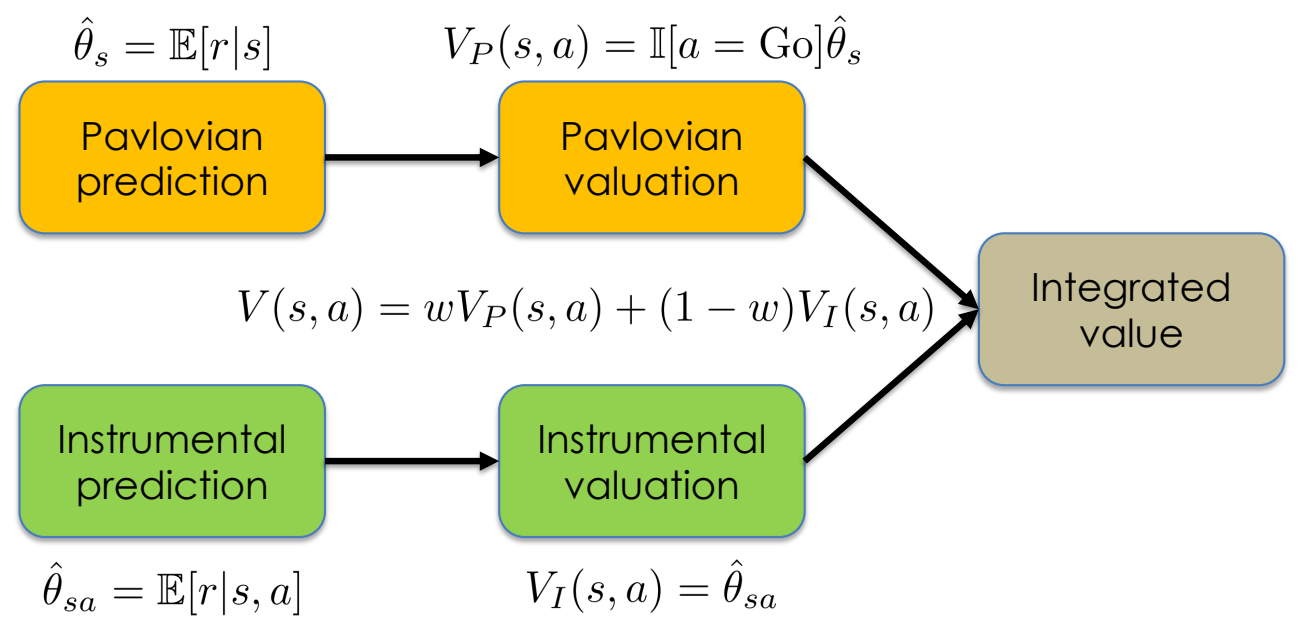

Figure 1: Experimental design and computational framework. (A) Shown here is the experimental design used by [20] in their EEG study, which differed in several minor ways from the design used by [21] in their fMRI study (see Materials and Methods). Subjects were instructed to respond to a target stimulus (white circle) by either pressing a button (Go) or witholding a button press (NoGo). Subjects had to learn the optimal action based on stimulus cues (shapes) and reward or punishment feedback. For all conditions, the optimal action yielded reward delivery or punishment avoidance with $70 \%$ probability; this probability was $30 \%$ for the suboptimal action. (B) Pavlovian and instrumental prediction and valuation combine into a single integrated decision value based on a weighting parameter $(w)$ that represents the evidence for the uncontrollable environment (i.e., in favor of the Pavlovian predictor). See Materials and Methods for technical details. 
to-Win, Go-to-Avoid, NoGo-to-Win, and NoGo-to-Avoid (Fig 1A). A key finding from this paradigm is that people make more errors on Go-to-Avoid trials compared to Go-to-Win trials, and this pattern reverses for NoGo trials, indicating that Pavlovian bias invigorates approach (the Go response) for reward-predictive cues, and inhibits approach for punishmentpredictive cues. By introducing decoy trials in which rewards were either controllable or uncontrollable, Dorfman and Gershman showed that the Pavlovian bias was enhanced in the low controllability condition (see also [26]).

An important innovation of the Dorfman and Gershman model was the hypothesis that the balance between Pavlovian and instrumental influences on action is dynamically arbitrated, and hence can potentially vary within the course of a single experimental session. This contrasts with most modeling of the Go/NoGo task (starting with [21]), which has assumed that the balance is fixed across the experimental session. Dorfman and Gershman presented behavioral evidence for within-session variation of the Pavlovian bias. Neural data could potentially provide even more direct evidence, by revealing correlates of the arbitration process itself. We pursue this question here by carrying out a model-based analysis of two prior data sets, one from an electroencephalography (EEG) study [20], and one from a functional magnetic resonance imaging (fMRI) study [21].

\section{Results}

\section{Modeling and behavioral results}

We fit computational models to Go/NoGo data from two previously published studies. The tasks used in these two studies were very similar, with a few minor differences detailed in the Materials and Methods. We will first briefly summarize the models (more details can be found in the Materials and Methods).

In [18], a Bayesian framework was introduced that formalized action valuation in terms of probabilistic inference (Fig 1B). According to this framework, Pavlovian and instrumental processes correspond to distinct predictive models of reward (or punishment) outcomes. The Pavlovian process estimates outcome predictions based on stimulus information alone, whereas the instrumental process uses both stimulus and action information. These predictions are converted into action values in different ways. For the instrumental process, action valuation is straightforward - it is simply the expected outcome for a particular stimulusaction pair. The Pavlovian process, which does not have an action-dependent outcome expectation, instead relies on the heuristic that reward-predictive cues should elicit behavioral approach (Go actions in the Go/NoGo task), and punishment-predictive cues should elicit avoidance (NoGo).

Arbitration in the Bayesian framework corresponds to model comparison: the action values are weighted by the probability favoring each predictor. This computation yields the expected action value under model uncertainty. Thus, the Bayesian framework offers an interpretation of Pavlovian bias in terms of the probability favoring Pavlovian outcome prediction (denoted by $w$, which we refer to as the "Pavlovian weight"). The Pavlovian 
weight can also be interpreted as the subjective degree of belief in an uncontrollable environment, where actions do not influence the probability distribution over outcomes (and correspondingly, $1-w$ is the degree of belief in a controllable environment).

Dorfman and Gershman [18] compared two versions of probabilistic arbitration. In the Fixed Bayesian model, the Pavlovian weight reflects a priori beliefs (i.e., prior to observing data). Thus, in the Fixed Bayesian model, the Pavlovian bias weight does not change with experience. In the Adaptive Bayesian model, the Pavlovian weight reflects a posteriori beliefs (i.e., after observing data), such that the weight changes across trials based on the observations. Finally, we compared both Bayesian models to a non-Bayesian reinforcement learning (RL) model that best described the data in [21]. This RL model is structurally similar to the Fixed Bayesian model, but posits a heuristic aggregation of Pavlovian and instrumental values. All models use an error-driven learning mechanism, but the Bayesian models assume that the learning rate decreases across stimulus repetitions.

We found that the Adaptive Bayesian model was favored in both data sets, with a protected exceedance probability greater than 0.7 (Fig 2A,B). To confirm that the Adaptive model fit the data well, we plotted the go bias (difference in accuracy between Go and NoGo trials) as a function of weight quantile (Fig 2C,D). Consistent with the model and previous results [18], the go bias increased with weight for the Win condition [top vs. bottom quantile: $t(31)=2.41, p<0.05$ for the EEG data set, $t(28)=3.96, p<0.001$ for the fMRI data set]. In contrast, it remained essentially flat for the Avoid condition. This asymmetry arises from the fact that most subjects were best fit with an initial Pavlovian value greater than $0(76 \%$ in the EEG data set, $63 \%$ in the fMRI data set). This means that the model actually predicts a positive go bias for the Avoid condition early during learning (when the Pavlovian weight is typically larger; see Fig S1), which eventually should become a negative go bias. Consistent with this hypothesis, the go bias during the first 40 trials (across all conditions) in the fMRI data set was significantly greater than 0 for the Avoid condition $[t(29)=3.63, p<0.002]$ and significantly less than 0 during the last 40 trials $[t(29)=2.24, p<0.05]$ (the EEG data set had fewer trials, and hence it was harder to obtain a reliable test of this hypothesis, though the results were numerically in the same direction).

In the next two sub-sections, we use the Adaptive model to generate model-based regressors for neural activity, in an effort to ground the hypothesized computational processes. In particular, we will focus on showing that neural signals covary with the Pavlovian weight, thereby demonstrating that this dynamically changing variable is encoded by the brain. Before proceeding to these analyses, it is important to show that this covariation is not confounded by other dynamic variables. In particular, while the Fixed and RL models lack a dynamic weight, the instrumental and Pavlovian values are dynamic in these models. To eliminate these variables as potential confounds, we correlated them with the Pavlovian weight for each subject. For both the EEG and fMRI data sets, the median correlation never exceeded 0.02 , and the median correlation never significantly differed fom $0(p>0.1$, signed rank test). This result gives us confidence that the neural covariation we report next is unconfounded by other dynamic variables in the computational model. 
A

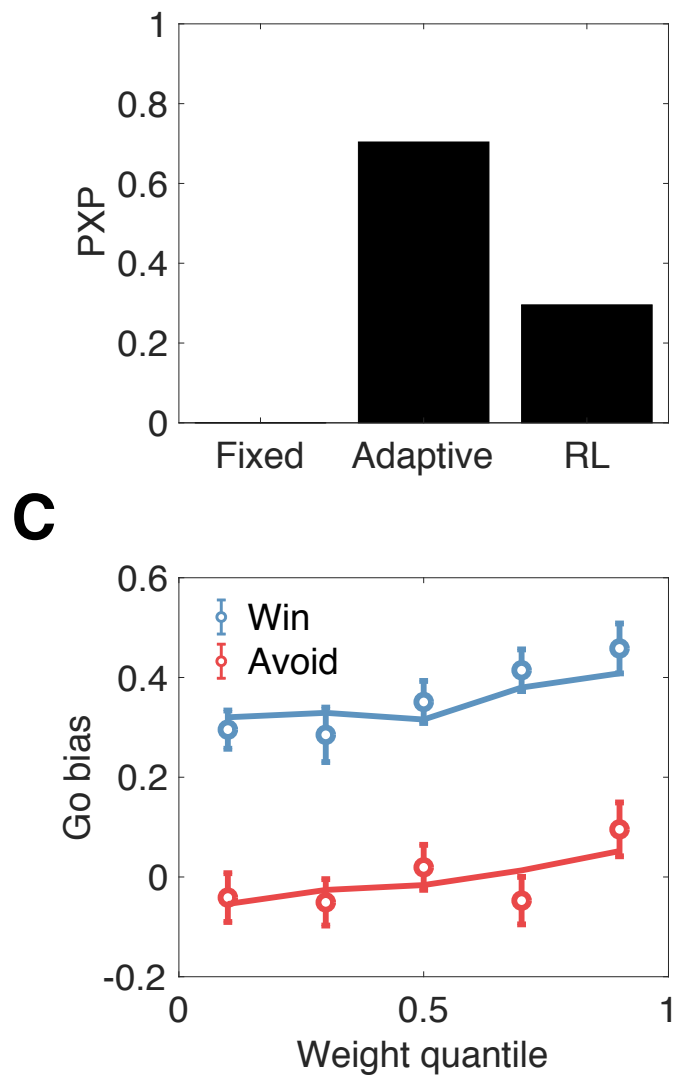

B
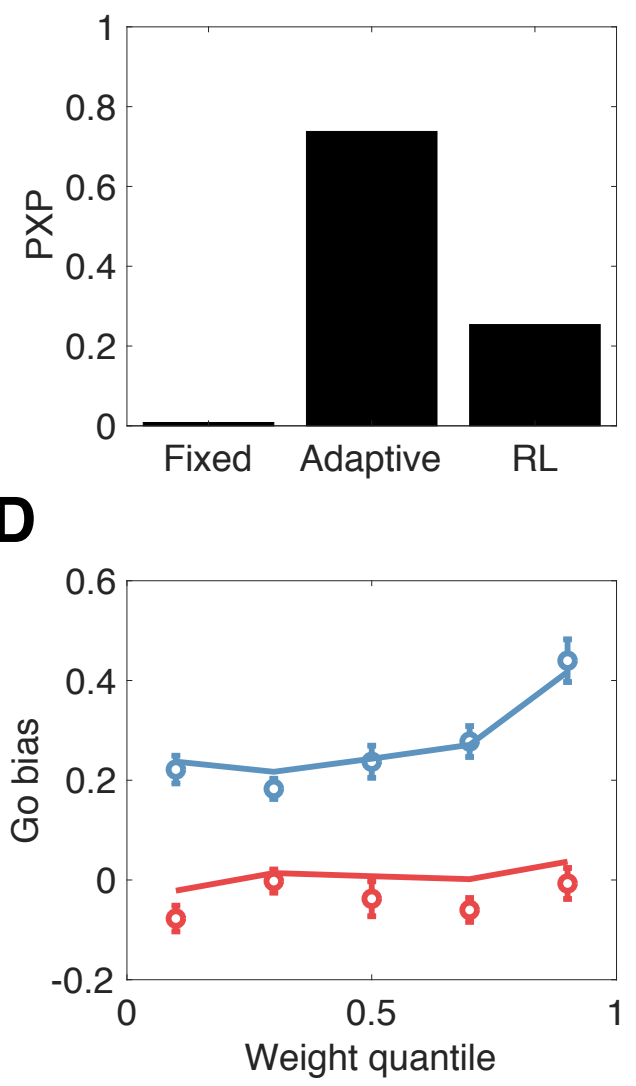

Figure 2: Behavioral results. Top: Protected exceedance probabilities (PXPs) for 3 computational models fit to the EEG data set (A) and the fMRI data set (B). Bottom: Go bias (difference in accuracy between Go and NoGo trials) computed as a function of the Pavlovian weight for the EEG data set (C) and the fMRI data set (D). Lines show model fits, circles show means with standard errors. 


\section{A}

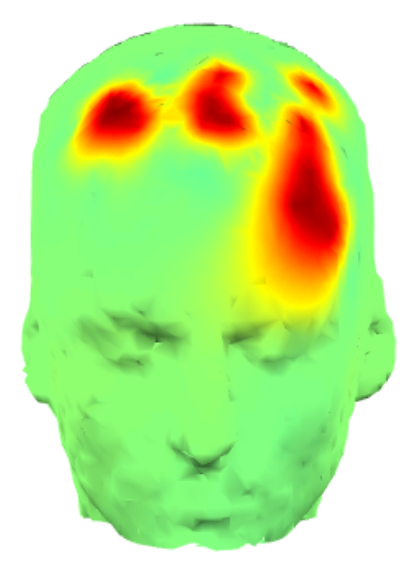

B

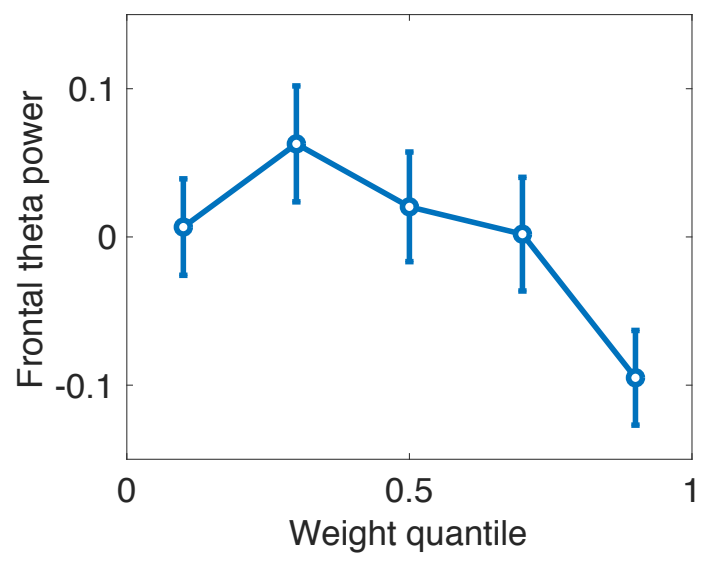

Figure 3: EEG results. (A) Montage showing region of interest, derived from [20]. (B) midfrontal theta power as a function of the Pavlovian weight. Error bars show standard error of the mean.

\section{EEG results}

Following the template of our behavioral analyses, we examined midfrontal theta power as a function of the Pavlovian weight (see Fig S3 for results fully disaggregated across conditions). In previous work on this same data set [20], and in follow-up studies [27, 26], frontal theta was implicated in the suppression of the Pavlovian influence on choice. Consistent with these previous findings, we found that frontal theta power decreased with the Pavlovian weight [top vs. bottom quantile: $t(31)=2.09, p<0.05$; Fig 3]. Unlike these earlier studies, which incorporated the frontal theta signal as an input into the computational model, we have validated for the first time a model of the frontal theta signal (i.e., as an output of the model).

\section{fMRI results}

We next re-analyzed fMRI data from [21], focusing on two frontal regions of interest: the inferior frontal gyrus (IFG; Fig 4A) and the ventromedial prefrontal cortex (vmPFC; Fig 4B). The key results are summarized in panels C and D in Fig 4 (see Fig S2 for fully disaggregated results, including results from the ventral striatum). When the Pavlovian weight is close to 0 , the IFG response for Go and NoGo conditions is not significantly different $(p=0.32)$, but when the Pavlovian weight is close to 1, IFG responds significantly more to NoGo than to Go $[t(28)=3.91, p<0.001$, Fig $4 \mathrm{C}]$. This is consistent with the hypothesis that IFG is responsible for the suppression of Go responses when the Pavlovian bias is strong, regardless of valence. Note that the NoGo¿Go effect is unsurprising given that the IFG region of interest was selected based on the NoGo¿Go contrast, but this selection criterion does not by itself 

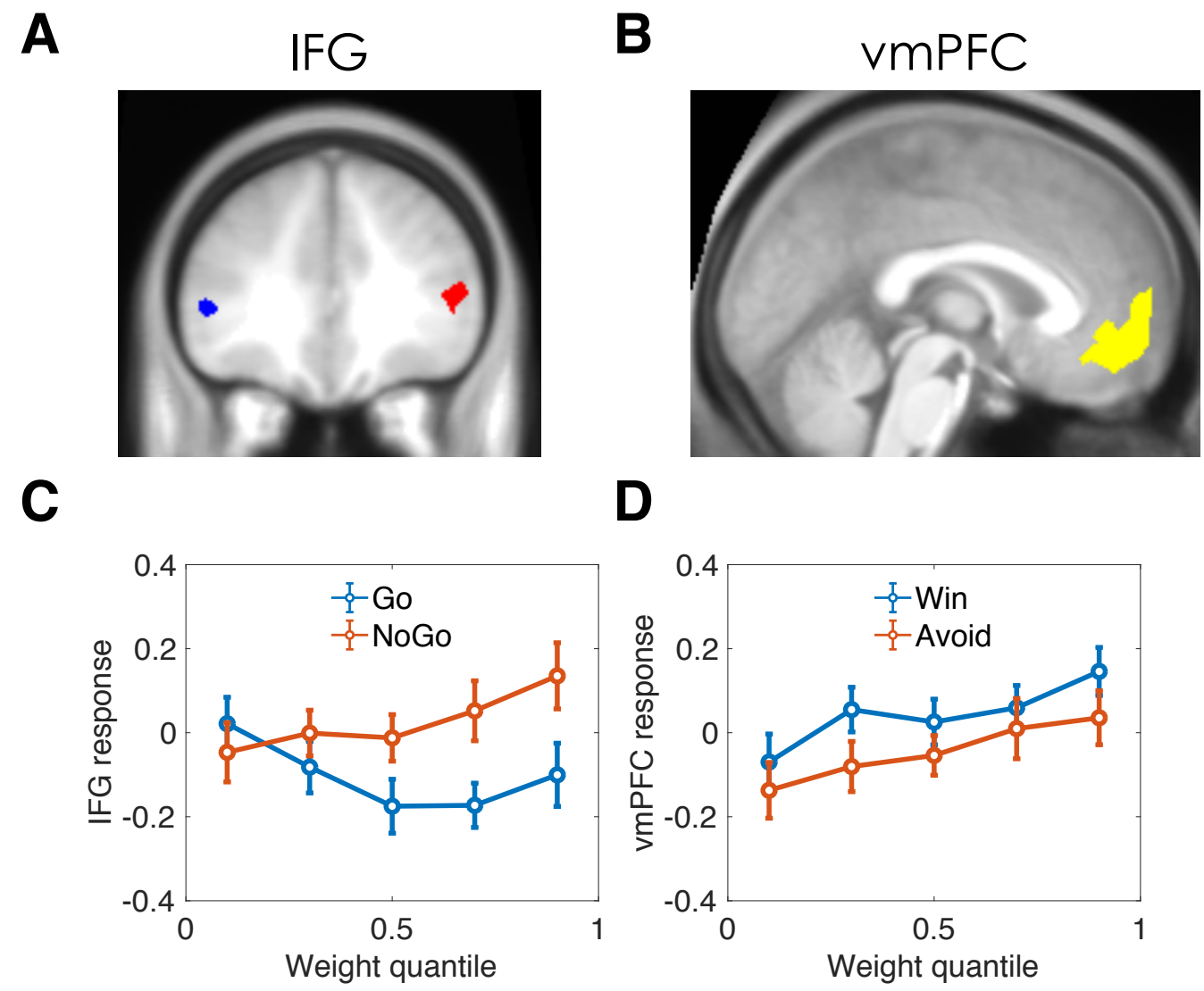

Figure 4: Functional MRI results. (A,B) Regions of interest. IFG: infeior frontal gyrus; vmPFC: ventromedial prefrontal cortex. (C) IFG response as a function of Pavlovian weight, separated by Go and NoGo conditions. (D) vmPFC response as a function of Pavlovian weight, separated by Win and Avoid conditions.

explain the interaction between weight and NoGo vs. Go.

The vmPFC scaled with the Pavlovian weight [top vs. bottom quantile: $t(28)=2.05, p<$ 0.05; Fig 4D], and responded more to Win vs. Avoid across weight quantiles $[t(28)=$ $3.46, p<0.002]$, but the interaction was not significant $(p=0.56)$. Thus, vmPFC appears to encode a combination of valence and Pavlovian bias (both main effects but no interaction).

\section{Discussion}

By re-analyzing two existing neuroimaging data sets, we have provided some of the first evidence for neural signals tracking beliefs about controllability during Go/NoGo task performance. These signals are theoretically significant, as they support the computational hypothesis that Pavlovian influences on choice behavior arise from a form of Bayesian model comparison between Pavlovian and instrumental outcome predictions [18]. Modeling of be- 
havior further supported this hypothesis, showing that the behavioral data were best explained by a Bayesian model in which Pavlovian influence changes as a function of inferred controllability.

Our analyses focused on three regions, based on prior research. One strong point of our approach is that we did not select the regions of interest based on any of the analyses reported in this paper; thus, the results serve as relatively unbiased tests of our computational hypotheses.

First, we showed that midfrontal theta power tracked inferred controllability (i.e., inversely with the Pavlovian weight). This finding is consistent with the original report describing the data set [20], which showed that the Pavlovian weight governing action selection could be partially predicted from midfrontal theta power, a finding further supported by subsequent research [27]. A recent study [26] attempted to more directly link midfrontal theta to controllability using a "learned helplessness" design in which one group of subjects intermittently lost control over outcomes by "yoking" the outcomes to those observed by a control group. The control group exhibited the same relationship between Pavlovian weight and midfrontal theta observed in earlier studies, whereas the yoked group did not (however, it must be noted that a direct comparison did not yield strong evidence for group differences). More broadly, these results are consistent with the hypothesis that midfrontal theta (and its putative cortical generator in midcingulate / dorsal anterior cingulate cortex) is responsible for computing the "need for control" [28] or the "expected value of control" [29]. Controllability is a necessary (though not sufficient) requirement for the exertion of cognitive control to have positive value.

Because of the partial volume acquisition in the imaging procedure (see Materials and Methods), our fMRI data did not allow us to examine hemodynamic correlates in the midcingulate cortex. Instead, we examined two other regions of interest: IFG and vmPFC. IFG has been consistently linked to inhibition of prepotent responses [30, 31]. Accordingly, we found greater response to NoGo than to Go in IFG. However, this difference only emerged when inferred controllability (as determined by our computational model) was low. There is some previous evidence that IFG is sensitive to controllability. Romaniuk and colleagues [32] reported that the IFG response was stronger on free choice trials compared to forced choice trials, and was significantly correlated with self-reported ratings of personal autonomy. Similarly, IFG activity has been associated with illusions of control [33]. It is difficult to directly connect these previous findings with those reported here, since the studies did not compare Go and NoGo responses.

While our finding that vmPFC shows a stronger response to reward vs. punishment is consistent with previous findings [34, 35], the fact that vmPFC decreases with inferred controllability is rather surprising. If anything, the literature suggests that vmPFC increases with subjective and objective controllability [36, 37, 38], though at least one study found a greater reduction in vmPFC activity after a controllable punishment compared to an uncontrollable punishment [39]. Further investigation is needed to confirm the surprising inverse relationship between vmPFC and inferred controllability.

Our study is limited in a number of ways, which point toward promising directions for 
future research. First, as already mentioned, our fMRI data did not allow us to test the hypothesis that midcingulate cortex, as the putative generator of midfrontal theta, tracked inferred controllability. This limitation could be overcome in future studies using whole brain acquisition volumes. Second, we only analyzed neural data timelocked to the stimulus; future work could examine outcome-related activity. We chose not to do this because of our focus on action selection, and in particular how inferred controllabiltiy signals are related to Pavlovian biasing of actions. An important task for future work will be to identify the neural update signal for inferred controllability that drives dynamic changes in Pavlovian bias.

\section{Materials and Methods}

This section summarizes the methods used in the original studies [21, 20], which can be consulted for further details. The Bayesian models were first presented in [18], and that paper can be consulted for derivations of the equations.

\section{Subjects}

30 adults (18-34 years) participated in the EEG study [20], and 47 adults (18-35 years) participated in the fMRI study [21]. Subjects had normal or corrected-to-normal vision, and no history of neurological, psychiatric, or other relevant medical problem. All subjects provided written informed consent, which was approved by the local ethics committees.

\section{Experimental procedure}

The experimental procedure was very similar across the two studies (see Fig 1A). Each trial began with a presentation of a visual stimulus (a colored shape in [20], a fractal in [21]) for $1000 \mathrm{~ms}$. After a variable interval (250-2500 $\mathrm{ms}$ in [20], 250-2000 ms in [21]), a target circle appeared, at which point a response was elicited. In [20], the target appeared centrally and subjects simply decided whether or not to press a button (Go or NoGo); in [21], the target appeared laterally and subjects (if they chose to respond) indicated on which side of the screen the target appeared. After a $1000 \mathrm{~ms}$ delay, subjects received reward or punishment feedback. In [20], the optimal action yielded a positive outcome (reward delivery or punishment avoidance) with probability 0.7 , and the suboptimal action yielded a positive outcome with probability 0.3 ; in [21] these probabilities were 0.8 and 0.2 , respectively. Rewards were defined as monetary gains, and punishments were defined as monetary losses. Subjects were compensated based on their earnings/losses during the task.

There were 4 conditions, signaled by distinct stimuli: Go-to-Win reward, Go-to-Avoid punishment, NoGo-to-Win reward, NoGo-to-Avoid punishment. Note that subjects were not instructed about the meaning of the stimuli, so these contingencies needed to be learned from trial and error. The experimental session consisted of 40 trials for each condition in [20], 60 trials for each condition in [21]. 


\section{EEG methods}

EEG was recorded using a 128-channel EGI system, recorded continuously with hardware filters set from 0.1 to $100 \mathrm{~Hz}$, a sampling rate of $250 \mathrm{~Hz}$, and an online vertex reference. The EEG data were then preprocessed to interpolate bad channels, remove eyeblink contaminants, and bandpass filtered. Finally, spectral power was computed within the theta band (4-8 Hz, 175-350 ms post-stimulus) in a midfrontal region of interest (ROI; Fig 4A) based on previous studies [40].

\section{fMRI methods}

Data were collected using a 3-Tesla Siemens Allegra magnetic resonance scanner (Siemens, Erlangen, Germany) with echo planar imaging of a partial volume that included the striatum and the midbrain (matrix: $128 \times 128$; 40 oblique axial slices per volume angled at $-30^{\circ}$ in the antero-posterior axis; spatial resolution: $1.5 \times 1.5 \times 1.5 \mathrm{~mm}$; TR $=4100 \mathrm{~ms}$; TE $=30 \mathrm{~ms}$ ). This partial volume included the whole striatum, the substantia nigra, ventral tegmental area, the amygdala, and the ventromedial prefrontal cortex. It excluded the medial cingulate cortex, the supplementary motor areas, the superior frontal gyrus, and the middle frontal gyrus. The fMRI acquisition protocol was optimized to reduce susceptibility-induced BOLD sensitivity losses in inferior frontal and temporal lobe regions [41].

Data were preprocessed using SPM8 (Wellcome Trust Centre for Neuroimaging, UCL, London), with the following steps: realignment, unwrapping using individual fieldmaps, spatial normalization to the Montreal Neurology Institute (MNI) space, smoothing with a $6 \mathrm{~mm}$ full-width half maximum Gaussian kernel, temporal filtering (high-pass cutoff: 128 $\mathrm{Hz}$ ), and whitened using a first-order autoregressive model. Finally, cue-evoked response amplitude was estimated with a general linear model (GLM), in which the event-related impulse was convolved with the canonical hemodynamic response function. The GLM also included movement regressors estimated from the realignment step.

To obtain a trial-by-trial estimate of the BOLD response at the time of the cue, we built a new GLM that included one regressor per trial at the time each cue was presented. In order to control for activity associated with the performance of the target detection task, we included a single regressor indicating the time at which the targets were presented together with a parametric modulator indicating whether participants performed a Go (1) or a NoGo $(-1)$ response. Similarly, to control for activity associated with the receipt of feedback, we included a single regressor indicating the time at which the outcome was presented together with a parametric modulator indicating whether the outcome was a loss $(-1)$, a neutral outcome (0), or a win (1). Finally, the model also included movement regressor parameters. Before estimation, all regressors were convolved with the canonical hemodynamic response function. This analysis resulted on one image per trial summarizing the BOLD response on that trial for each available voxel. We then extracted the mean BOLD response with the 2 frontal ROIs. The IFG ROI was defined as the voxels that responded to NoGo¿Go in learners in the original report, thresholded at $p<0.001$ uncorrected. The vmPFC ROI was defined as the voxels that responded positively to the parametric modulator of outcome 
responses in the GLM reported above, thresholded at $p<0.001$ uncorrected.

\section{Computational models}

We compared three computational models of learning and choice. Each model was fit to data from individual subjects using maximum likelihood estimation and compared using randomeffects Bayesian model comparison with the Bayesian information criterion approximation of the marginal likelihood [42]. We summarize the model comparison results using protected exceedance probabilities, which express the posterior probability that a particular model is more frequent in the population than all other models, adjusting for the probability that the differences in model fit could have arisen from the null hypothesis (uniform model frequency in the population).

Guitart-Masip and colleagues [21] compared several reinforcement learning models, finding the strongest support for one in which the action policy is defined by:

$$
P(\mathrm{Go} \mid s)=\frac{\exp [V(s, \mathrm{Go})]}{\exp [V(s, \mathrm{Go})]+\exp [V(s, \mathrm{NoGo})]}(1-\xi)+\frac{\xi}{2},
$$

where $s$ denotes the stimulus, $\xi$ is a lapse probability (capturing a baseline error rate), and $V(s, a)$ is the integrated action value for action $a$ in response to stimulus $s$ :

$$
\begin{aligned}
& V(s, \mathrm{Go})=V_{I}(s, \mathrm{Go})+\pi V_{P}(s, \mathrm{Go})+b \\
& V(s, \mathrm{NoGo})=V_{I}(s, \mathrm{NoGo}) .
\end{aligned}
$$

The action value integrates the instrumental value $V_{I}$ and the Pavlovian value $V_{P}$, where the weighting parameter $\pi$ captures a fixed Pavlovian approach bias towards reward-predictive cues, and an avoidance bias away from punishment predictive cues. In addition, the parameter $b$ captures a fixed Go bias. The values are updated according to an error-driven learning rule:

$$
\begin{aligned}
& \Delta V_{I}(s, a)=\alpha\left[\rho r-V_{I}(s, a)\right] \\
& \Delta V_{P}(s)=\alpha\left[\rho r-V_{P}(s)\right],
\end{aligned}
$$

where $\alpha$ is a learning rate, $\rho>0$ is an outcome scaling factor, and $r$ is the outcome. For the sake of brevity, we will refer to this model simply as the "RL model" (but note that the models described next could be validly considered "Bayesian RL models" insofar as they estimate expectations about reward and punishment; see [43] for further discussion of this point).

Subsequent modeling (e.g., [20]) has shown that this model can be improved by allowing differential sensitivity to rewards and punishments, but we do not pursue that extension here since it would also require us to develop an equivalent extension of the Bayesian models described next. Since our primary goal is to model the neural dynamics underlying variability in the Pavlovian bias, we did not feel that it was necessary to run a more elaborate horse race between the model classes. 
Dorfman and Gershman [18] introduced two Bayesian models. The learner is modeled as occupying one of two possible environments (controllable or uncontrollable). In the controllable environment, outcomes depend on the combination of stimulus and action, as specified by a Bernoulli parameter $\theta_{s a}$. In the uncontrollable environment, ouctomes depend only on the stimulus, as specified by the parameter $\theta_{s}$. Because these parameters are unknown at the outset, the learner must estimate them. The Bayes-optimal estimate, assuming a Beta prior on the parameters, can be computed using an error-driven learning rule similar to the one described above, with the difference that the learning rate declines according to $\alpha=1 / \eta_{s}$ for the Pavlovian model, where $\eta_{s}$ is the number of times stimulus $s$ was encountered (the instrumental model follows the same idea, but using $\eta_{s a}$, the number of times action $a$ was taken in response to stimulus $s$ ). The model is parametrized by the initial value of $\eta$ and the initial values, which together define Beta distribution priors (see [18] for a complete derivation). To convert the parameter estimates (denoted $\hat{\theta}_{s}$ and $\hat{\theta}_{s a}$ ) into action values, we assumed that the instrumental values are simply the parameter estimates, $V_{I}(s, a)=\hat{\theta}_{s a}$, while the Pavlovian value $V_{P}$ is 0 for $a=$ NoGo and $\hat{\theta}_{s}$ for Go.

The learner does not know with certainty which environment she occupies; her belief that she is in the controllable environment is specified by the probability $w$. The expected action value under environment uncertainty is then given by:

$$
V(s, \mathrm{Go})=(1-w) V_{I}(s, \mathrm{Go})+w V_{P}(s, \mathrm{Go}),
$$

which is similar to the RL model integration but where the integrated action value is now a convex combination of the instrumental and Pavlovian values. Unlike the RL model, the Fixed Bayesian model used an inverse temperature parameter instead of an outcome scaling parameter (though these parameters play essentially the same role), and did not model lapse probability or Go bias (because the extra complexity introduced by these parameters was not justified based on model comparison). Thus, the action policy is given by:

$$
P(\mathrm{Go} \mid s)=\frac{\exp [\beta V(s, \mathrm{Go})]}{\exp [\beta V(s, \mathrm{Go})]+\exp [\beta V(s, \text { NoGo })]},
$$

where $\beta$ is the inverse temperature, which controls action stochasticity.

In the Bayesian framework, the parameter $w$ can be interpreted as a belief in the probability that the environment is uncontrollable (outcomes do not depend on actions). A critical property of the Fixed Bayesian model is that this parameter is fixed for a subject, under the assumption that the subject does not draw inferences about controllability during the experimental session. The Adaptive Bayesian model is essentially the same as the Fixed Bayesian model, but departs in one critical aspect: the Pavlovian weight parameter $w$ is updated on each trial. Using the relation $w=1 /(1+\exp (-L))$, where $L$ is the $\log$-odds favoring the uncontrollable environment, we can describe the update rule as follows:

$$
\Delta L=r \log \frac{\hat{\theta}_{s}}{\hat{\theta}_{s a}}+(1-r) \frac{1-\hat{\theta}_{s}}{1-\hat{\theta}_{s a}}
$$

The initial value of $L$ was set to 0 (a uniform distribution over environments). 


\section{Code and data availability}

All code and data for reproducing the analyses and figures is available at https://github . com/sjgershm/GoNoGo-neural.

\section{Acknowledgments}

SJG was supported by the Center for Brains, Minds and Machines (CBMM), funded by NSF STC award CCF-1231216, and by the Office of Naval Research (N00014-17-1-2984). MGM was supported by a research grant awarded by the Swedish Research Council (VR-201802606). JFC was supported by NIMH 1RO1MH119382-01.

\section{References}

[1] Breland K, Breland M. The misbehavior of organisms. American Psychologist. 1961;16:681-684.

[2] Dayan P, Niv Y, Seymour B, Daw ND. The misbehavior of value and the discipline of the will. Neural Networks. 2006;19:1153-1160.

[3] Williams DR, Williams H. Auto-maintenance in the pigeon: sustained pecking despite contingent non-reinforcement. Journal of the experimental analysis of behavior. $1969 ; 12: 511-520$.

[4] Hershberger WA. An approach through the looking-glass. Animal Learning \& Behavior. 1986;14:443-451.

[5] Grossen N, Kostansek D, Bolles R. Effects of appetitive discriminative stimuli on avoidance behavior. Journal of Experimental Psychology. 1969;81:340-343.

[6] Bull III JA. An interaction between appetitive Pavlovian CSs and instrumental avoidance responding. Learning and Motivation. 1970;1:18-26.

[7] Estes W, Skinner B. Some quantitative properties of anxiety. Journal of Experimental Psychology. 1941;29:390-400.

[8] Annau Z, Kamin L. The conditioned emotional response as a function of intensity of the US. Journal of Comparative and Physiological Psychology. 1961;54:428-432.

[9] Huys QJ, Eshel N, O'Nions E, Sheridan L, Dayan P, Roiser JP. Bonsai trees in your head: how the pavlovian system sculpts goal-directed choices by pruning decision trees. PLoS Computational Biology. 2012;8.

[10] Rescorla R, Solomon R. Two-process learning theory: relationships between Pavlovian conditioning and instrumental learning. Psychological Review. 1967;74:151-182. 
[11] Guitart-Masip M, Duzel E, Dolan R, Dayan P. Action versus valence in decision making. Trends in Cognitive Sciences. 2014;18:194-202.

[12] Joel D, Niv Y, Ruppin E. Actor-critic models of the basal ganglia: New anatomical and computational perspectives. Neural Networks. 2002;15:535-547.

[13] O’Doherty J, Dayan P, Schultz J, Deichmann R, Friston K, Dolan RJ. Dissociable roles of ventral and dorsal striatum in instrumental conditioning. Science. 2004;304:452-454.

[14] Daw ND, Niv Y, Dayan P. Uncertainty-based competition between prefrontal and dorsolateral striatal systems for behavioral control. Nature Neuroscience. 2005;8:17041711.

[15] Keramati M, Dezfouli A, Piray P. Speed/accuracy trade-off between the habitual and the goal-directed processes. PLoS Computational Biology. 2011;7.

[16] Lee SW, Shimojo S, O’Doherty JP. Neural computations underlying arbitration between model-based and model-free learning. Neuron. 2014;81:687-699.

[17] Kool W, Gershman SJ, Cushman FA. Cost-benefit arbitration between multiple reinforcement-learning systems. Psychological Science. 2017;28:1321-1333.

[18] Dorfman HM, Gershman SJ. Controllability governs the balance between Pavlovian and instrumental action selection. Nature Communications. 2019;10:1-8.

[19] Holmes NM, Marchand AR, Coutureau E. Pavlovian to instrumental transfer: a neurobehavioural perspective. Neuroscience \& Biobehavioral Reviews. 2010;34:1277-1295.

[20] Cavanagh JF, Eisenberg I, Guitart-Masip M, Huys Q, Frank MJ. Frontal theta overrides pavlovian learning biases. Journal of Neuroscience. 2013;33:8541-8548.

[21] Guitart-Masip M, Huys QJ, Fuentemilla L, Dayan P, Duzel E, Dolan RJ. Go and no-go learning in reward and punishment: interactions between affect and effect. NeuroImage. 2012;62:154-166.

[22] Crockett MJ, Clark L, Robbins TW. Reconciling the role of serotonin in behavioral inhibition and aversion: acute tryptophan depletion abolishes punishment-induced inhibition in humans. Journal of Neuroscience. 2009;29:11993-11999.

[23] Guitart-Masip M, Fuentemilla L, Bach DR, Huys QJ, Dayan P, Dolan RJ, et al. Action dominates valence in anticipatory representations in the human striatum and dopaminergic midbrain. Journal of Neuroscience. 2011;31:7867-7875.

[24] Crockett MJ, Clark L, Apergis-Schoute AM, Morein-Zamir S, Robbins TW. Serotonin modulates the effects of Pavlovian aversive predictions on response vigor. Neuropsychopharmacology. 2012;37:2244-2252. 
[25] de Boer L, Axelsson J, Chowdhury R, Riklund K, Dolan RJ, Nyberg L, et al. Dorsal striatal dopamine D1 receptor availability predicts an instrumental bias in action learning. Proceedings of the National Academy of Sciences. 2019;116:261-270.

[26] Csifcsák G, Melsæter E, Mittner M. Intermittent absence of control during reinforcement learning interferes with Pavlovian bias in action selection. Journal of Cognitive Neuroscience. 2020;32:646-663.

[27] Swart JC, Frank MJ, Määttä JI, Jensen O, Cools R, den Ouden HE. Frontal network dynamics reflect neurocomputational mechanisms for reducing maladaptive biases in motivated action. PLoS Biology. 2018;16:e2005979.

[28] Cavanagh JF, Frank MJ. Frontal theta as a mechanism for cognitive control. Trends in Cognitive Sciences. 2014;18:414-421.

[29] Shenhav A, Botvinick MM, Cohen JD. The expected value of control: an integrative theory of anterior cingulate cortex function. Neuron. 2013;79:217-240.

[30] Rubia K, Smith AB, Brammer MJ, Taylor E. Right inferior prefrontal cortex mediates response inhibition while mesial prefrontal cortex is responsible for error detection. NeuroImage. 2003;20:351-358.

[31] Aron AR, Poldrack RA. Cortical and subcortical contributions to stop signal response inhibition: role of the subthalamic nucleus. Journal of Neuroscience. 2006;26:2424-2433.

[32] Romaniuk L, Sandu AL, Waiter GD, McNeil CJ, Xueyi S, Harris MA, et al. The neurobiology of personal control during reward learning and its relationship to mood. Biological Psychiatry: Cognitive Neuroscience and Neuroimaging. 2019;4:190-199.

[33] Lorenz RC, Gleich T, Kühn S, Pöhland L, Pelz P, Wüstenberg T, et al. Subjective illusion of control modulates striatal reward anticipation in adolescence. NeuroImage. $2015 ; 117: 250-257$.

[34] Blair K, Marsh AA, Morton J, Vythilingam M, Jones M, Mondillo K, et al. Choosing the lesser of two evils, the better of two goods: specifying the roles of ventromedial prefrontal cortex and dorsal anterior cingulate in object choice. Journal of Neuroscience. 2006;26:11379-11386.

[35] Monosov IE, Hikosaka O. Regionally distinct processing of rewards and punishments by the primate ventromedial prefrontal cortex. Journal of Neuroscience. 2012;32:1031810330.

[36] Amat J, Baratta MV, Paul E, Bland ST, Watkins LR, Maier SF. Medial prefrontal cortex determines how stressor controllability affects behavior and dorsal raphe nucleus. Nature Neuroscience. 2005;8:365-371. 
[37] Kerr DL, McLaren DG, Mathy RM, Nitschke JB. Controllability modulates the anticipatory response in the human ventromedial prefrontal cortex. Frontiers in Psychology. $2012 ; 3: 557$.

[38] Murayama K, Matsumoto M, Izuma K, Sugiura A, Ryan RM, Deci EL, et al. How selfdetermined choice facilitates performance: A key role of the ventromedial prefrontal cortex. Cerebral Cortex. 2015;25:1241-1251.

[39] Bhanji JP, Delgado MR. Perceived control influences neural responses to setbacks and promotes persistence. Neuron. 2014;83:1369-1375.

[40] Cavanagh JF, Zambrano-Vazquez L, Allen JJ. Theta lingua franca: A common midfrontal substrate for action monitoring processes. Psychophysiology. 2012;49:220-238.

[41] Weiskopf N, Hutton C, Josephs O, Deichmann R. Optimal EPI parameters for reduction of susceptibility-induced BOLD sensitivity losses: a whole-brain analysis at $3 \mathrm{~T}$ and 1.5 T. NeuroImage. 2006;33:493-504.

[42] Rigoux L, Stephan KE, Friston KJ, Daunizeau J. Bayesian model selection for group studies - revisited. NeuroImage. 2014;84:971-985.

[43] Gershman SJ. A unifying probabilistic view of associative learning. PLoS Computational Biology. 2015;11. 


\section{Supplementary Figures}

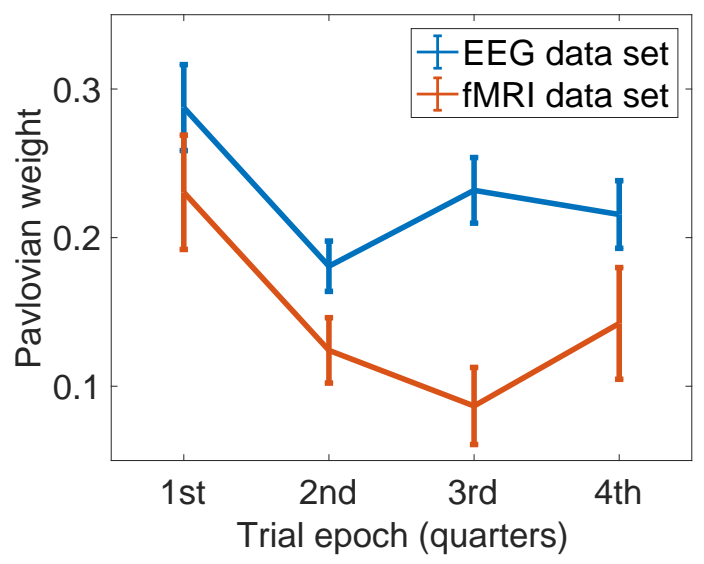

Figure S1: Pavlovian weight dynamics. The weight variable $w$ is plotted across trial epochs, broken into quarters (note that the data sets have different numbers of trials). Error bars show standard error of the mean. 


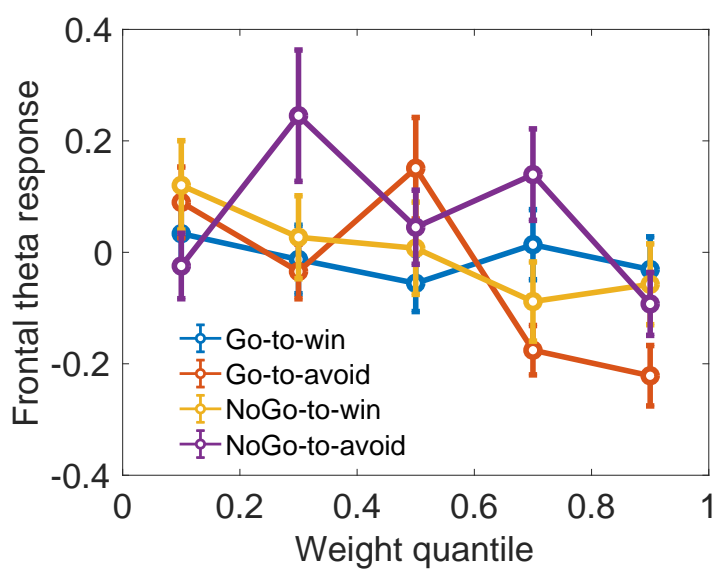

Figure S2: Disaggregated EEG results. Midfrontal theta power (z-scored within subject) as a function of Pavlovian weight quantile, separated by stimulus condition. Error bars show standard error of the mean.
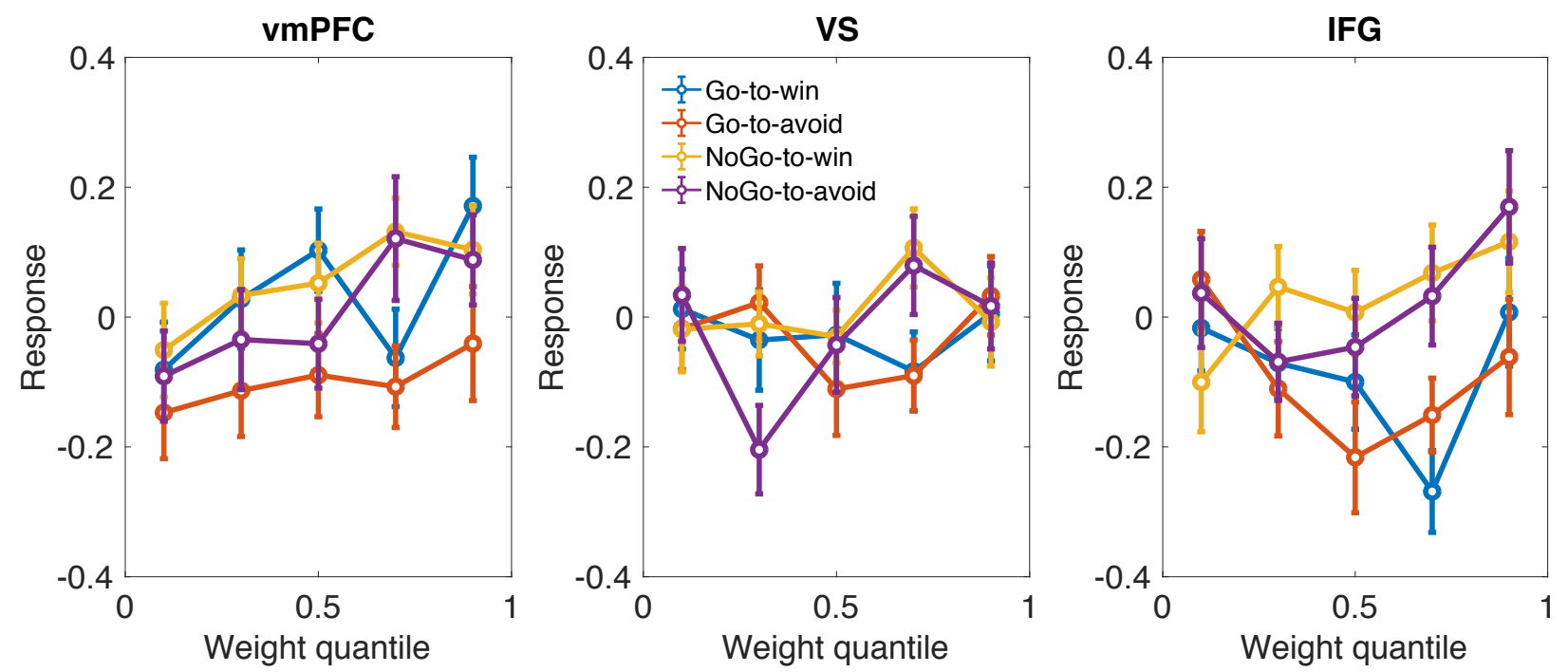

Figure S3: Disaggregated fMRI results. BOLD response amplitude (z-scored within subject) as a function of Pavlovian weight quantile, separated by stimulus condition. Left: ventromedial prefrontal cortex. Middle: ventral striatum. Right: inferior frontal gyrus. Error bars show standard error of the mean. 